\title{
The X-ray, optical and infrared counterpart to GRB 980703
}

\author{
P.M. Vreeswijk ${ }^{1}$, T.J. Galama ${ }^{1}$, A.N. Owens ${ }^{2}$, T. Oosterbroek $^{2}$, T. Geballe ${ }^{3}$, J. van Paradijs ${ }^{1,4}$, C. Kouveliotou ${ }^{5,6}$,

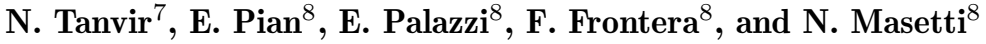 \\ 1 Astronomical Institute "Anton Pannekoek", University of Amsterdam, \& Center for High Energy Astrophysics, Kruislaan \\ 403, 1098 SJ Amsterdam, The Netherlands \\ 2 Astrophysics Division, Space Science Department of ESA, European Space Research and Technology Centre, 2200 AG \\ Noordwijk, The Netherlands \\ 3 Joint Astronomy Centre, 660 N. A'ohoku Place, Hilo, Hawaii 9672 0, U.S.A. \\ 4 Physics Department, University of Alabama in Huntsville, Huntsville AL 35899, U.S.A. \\ 5 Universities Space Research Association \\ 6 NASA/MSFC, Code ES-84, Huntsville AL 35812, U.S.A. \\ 7 Institute of Astronomy, Madingley Road, Cambridge CB3 OHA, UK \\ 8 Istituto Tecnologie e Studio Radiazioni Extraterrestri (TESRE), CNR, Via P. Gobetti 101, 40129 Bologna, Italy
}

Received December 29, 1998; accepted March 10, 1999

\begin{abstract}
We report on X-ray, optical and infrared followup observations of GRB 980703. We detect a previously unknown X-ray source in the GRB error box; assuming a power law decline we find for its decay index $\alpha<$ $-0.91(3 \sigma)$. We invoke host galaxy extinction to match the observed spectral slope with the slope expected from "fireball" models. We find no evidence for a spectral break in the infrared to X-ray spectral range on 1998 July 4.4, and determine a lower limit of the cooling break frequency: $\nu_{\mathrm{c}}>1.310^{17} \mathrm{~Hz}$. For this epoch we obtain an extinction of $A_{V}=1.50 \pm 0.11$. From the X-ray data we estimate the optical extinction to be $A_{V}=20.2_{-7.3}^{+12.3}$, inconsistent with the former value. Following Wijers \& Galama (1998), we determine intrinsic fireball properties for this burst.
\end{abstract}

Key words: gamma-rays: bursts — radiation mechanisms: non-thermal

\section{Introduction}

GRB 980703 was detected on July 3.182 UT with the All Sky Monitor (ASM) on the Rossi X-ray Timing Explorer (RXTE; Levine et al. 1998), the Burst And Transient Source Experiment (BATSE, trigger No. 6891; Kippen et al. 1998), Beppo SAX (Amati et al. 1998) and Ulysses (Hurley et al. 1998).

\section{X-ray observations}

We observed the ASM error box of GRB 980703 with the BeppoSAX LECS $(0.1-10 \mathrm{keV})$ and MECS $(2-10 \mathrm{keV})$ on July 4.10-5.08 UT and July 7.788.71 UT. These data show a previously unknown X-ray source 1SAX J2359.1+0835 at RA $=23^{\mathrm{h}} 59^{\mathrm{m}} 06.8$, Dec $=+08^{\circ} 35^{\prime} 45^{\prime \prime}$ (equinox J2000.0), with an error radius of $50^{\prime \prime}$. The average spectrum can be modelled with a photon index $\Gamma=2.51 \pm 0.32$ and a host galaxy column density $N_{\mathrm{H}}$ (host) $=3.6_{-1.3}^{+2.2} \quad 10^{22} \mathrm{~cm}^{-2}$, corresponding to $A_{V}$ (host) $=20.2_{-7.3}^{+12.3}$ (local to the absorber).

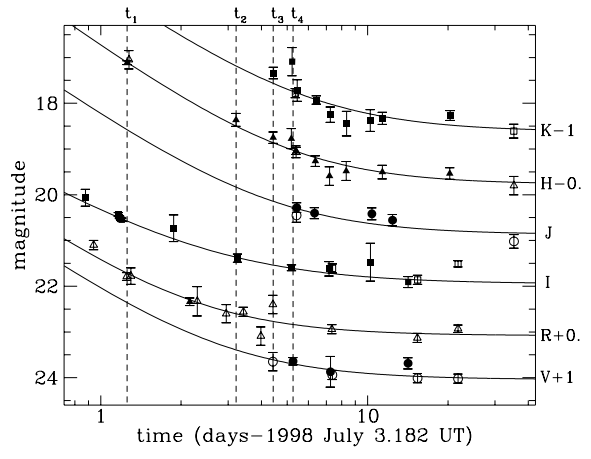

Fig. 1. $V, R, I, J, H$ and $K$ light curves of GRB 980703. The filled symbols denote our data, while the open symbols represent data taken from the literature (see Vreeswijk et al. 1998 for the references). At the epochs $t_{1}-t_{4}$ we have reconstructed the spectral flux distribution of the OT

Fitting a power law model to the light curve, we obtain $\alpha<-0.91(3 \sigma)$ for the decay index. We checked for the presence of the Fe line at $6.4 \mathrm{keV}$, but do not detect it. The upper limit on its flux is $8.310^{-6}$ photons $\mathrm{cm}^{-2} \mathrm{~s}^{-1}$ (90\% confidence level).

\section{Optical and infrared observations}

We observed the field of GRB 980703 with the Wise Observatory $1-\mathrm{m}$ telescope (in $I$ ); the $3.5-\mathrm{m}$ New Technology Telescope (NTT; in $V, I$ and $H$ ), the $2.2-\mathrm{m}$ (in $H$ and $K s$ ) and Dutch 90-cm (in gunn $i$ ) telescopes of ESO (La Silla); the CTIO 0.9-m telescope (in $R$ ) and UKIRT (in $H, J$ and $K$ ).

The light curves of the OT, which was independently discovered by Frail et al. (1998) and Zapatero Osorio et al. (1998), are shown in Fig. 1 (see Vreeswijk et al. for the photometric calibration and a log of the observations). In view of the flattening of the light curves after $t \sim 5$ days we fitted a model $F_{\nu}=F_{0} \cdot t^{\alpha}+F_{\text {gal }}$ to our own $I$ and $H$ band light curves. The weighted mean value of $\alpha$ for the 


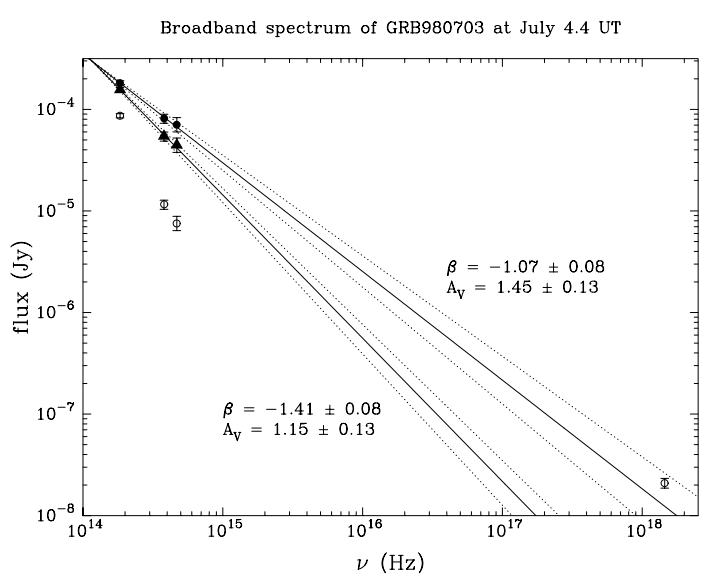

Fig. 2. Broad-band spectrum of GRB 980703 at July 4.4 UT (i.e., at $t_{1}$ in Fig. 1). The open symbols are the $R, I$ and $H$ OT fluxes (interpolated to July 4.4, corrected for Galactic foreground absorption and the host galaxy flux) and the MECS $(2-10 \mathrm{keV})$ de-absorbed flux. The filled symbols are obtained by invoking an interstellar extinction, $A_{V}$, to force the slope of the data points to take on the two possible theoretical spectral slopes. The two slopes $\beta$ and their $1 \sigma$ errors are indicated by the solid and dotted lines

$I$ and $H$-bands equals $-1.61 \pm 0.12$. For the $V, R, J$ and $K$ bands we fixed $\alpha$ at this value, included also data from the literature, and only fitted $m_{0}$ and $m_{\text {gal }}$. The fits are shown as solid lines in Fig. 1. For the first epoch $\left(t_{1}\right)$ we fitted the resulting spectral flux distribution with a power law, $F_{\nu} \propto \nu^{\beta}$, and find $\beta=-2.71 \pm 0.12$.

\section{Discussion}

If we make the assumption that the OT emission is due to synchrotron radiation from electrons with a power law energy distribution (with index $p$ ), one expects a relation between $p$, the spectral slope $\beta$, and the decay constant $\alpha$ (Sari et al. 1998). One must distinguish two cases: (i) both the peak frequency $\nu_{\mathrm{m}}$ and the cooling frequency $\nu_{\mathrm{c}}$ are below the optical/IR waveband. Then $p=$ $(-4 \alpha+2) / 3=2.81 \pm 0.16$ and $\beta=-p / 2=-1.41 \pm 0.08$, (ii) $\nu_{\mathrm{m}}$ has passed the optical/IR waveband, but $\nu_{\mathrm{c}}$ has not yet. In that case $p=(-4 \alpha+3) / 3=3.15 \pm 0.16$ and $\beta=-(p-1) / 2=-1.07 \pm 0.08$. In both cases the expected value of $\beta$ is inconsistent with the observed $\beta=-2.71 \pm 0.12$. Following Ramaprakash et al. (1998) we assume that the discrepancy is caused by host galaxy extinction. To determine the host galaxy absorption we first blueshifted the OT flux distribution to the host galaxy rest frame (using $z=0.966$ determined by Djorgovski et al. 1998), and then applied an extinction correction using the Galactic extinction curve of Cardelli et al. (1989), to obtain the expected spectral slope $\beta$. For epoch $t_{1}$ (July $4.4 \mathrm{UT}$ ), we obtain $A_{V}=1.15 \pm 0.13$ and $A_{V}=1.45 \pm 0.13$ for the cases (i) and (ii), respectively (see Fig. 2). In case (i) we find that an extrapolation of the optical flux distribution to higher frequencies predicts an X-ray flux that is significantly below the observed value, whereas in case (ii) the extrapolated and observed values are in excellent agreement. The mismatch in case (i) is into a direction that cannot be interpreted in terms of evidence for a cooling break between the optical and X-ray wavebands. When we include the X-ray data point in the fit to obtain a more accurate determination of $A_{V}$, we find $A_{V}=1.50 \pm 0.11$, and $\beta=-1.013 \pm 0.016$. We estimate the $(2 \sigma)$ lower limit to the cooling frequency to be $\nu_{\mathrm{c}}>1.310^{17} \mathrm{~Hz}$ $\left(\nu_{\mathrm{c}}>0.5 \mathrm{keV}\right)$.

We performed the same analysis for the other epoch $\left(t_{4}\right)$ with X-ray data. At this epoch, the X-ray upper limit does not allow us to discriminate between the two cases. However, we can still estimate a lower limit to the cooling break from its time dependence: $\nu_{\mathrm{c}} \propto t^{-1 / 2}$, which would allow the break to drop to $\nu_{\mathrm{c}}>6.310^{16} \mathrm{~Hz}$ only, between epoch $t_{1}$ and $t_{4}$. On the basis of our analysis we conclude that there is no strong evidence for a cooling break between the optical/IR and the $2-10 \mathrm{keV}$ passband before 1998 July 8.4 UT. This conclusion is at variance with the inference of Bloom et al. (1998). For the latter epoch we obtain $A_{V}=0.86 \pm 0.25$ and $\beta=-0.90 \pm 0.06$, after inclusion of the X-ray point. On the basis of this analysis we conclude that there is no evidence for a cooling break between the optical/IR and the $2-10 \mathrm{keV}$ passband before 1998 July $8.8 \mathrm{UT}$. This conclusion is at variance with that of Bloom et al. (1998).

Following the analysis of Wijers \& Galama (1998) we have determined the following intrinsic fireball properties: (i) the energy of the blast wave per unit solid angle: $\mathcal{E}>510^{52} \mathrm{erg} /(4 \pi$ sterad), (ii) the ambient density: $n>1.1$ nucleons $\mathrm{cm}^{-3}$, (iii) the percentage of the nucleon energy density in electrons: $\epsilon_{\mathrm{e}}>0.13$, and (iv) in the magnetic field: $\epsilon_{B}<610^{-5}$. The very low energy in the magnetic field, $\epsilon_{B}$, is a natural reflection of the high frequency of the cooling break $\nu_{\mathrm{c}}$ (see Vreeswijk et al. 1998).

\section{References}

Amati L., et al., 1998, GCN Circ., 146 Bloom J.S., et al., 1998, ApJ 508, L21

Cardelli J.A., et al., 1989, ApJ 345, 245

Djorgovski S.G., et al., 1998, ApJ 508, L17

Frail D.A., et al., 1998, GCN Circ., 128

Galama T.J., et al., 1998, GCN Circ., 127

Hurley K., et al., 1998, GCN Circ., 125

Kippen M., et al., 1998, GCN Circ., 143

Levine A., et al., 1998, IAU Circ., 6966

Predehl P., Schmitt J.H.M.M., 1995, A\&A 293, 889

Sari R., Piran T., Narayan R., 1998, ApJ 497, L17

Ramaprakash A.N., et al., 1998, Nat 393, 43

Schlegel D.J., et al., 1998, ApJ 500, 525

Vreeswijk P.M., et al., 1998, ApJ (in press)

Wijers R.A.M.J., Galama T.J., 1998, ApJ (in press) Zapatero Osorio M.R., et al., 1998, IAU Circ., 6967 